\title{
Brain Perfusion and Astrocytes
}

\author{
Bruno Cauli ${ }^{1 *}$ and Edith $\mathrm{Hamel}^{2 *}$
}

${ }^{1}$ Sorbonne Universités, UPMC Univ Paris 06, INSERM, CNRS, Neuroscience Paris Seine Institut de Biologie Paris Seine (NPS - IBPS), 75005 Paris, France and ${ }^{2}$ Laboratory of Cerebrovascular Research, Montreal Neurological Institute, McGill University, Montréal, QC, Canada, H3A 2B4

\section{*Correspondence:}

Bruno Cauli:

Sorbonne Universités, UPMC Univ Paris 06, INSERM, CNRS, Neuroscience Paris Seine Institut de Biologie Paris Seine (NPS - IBPS), 75005 Paris, France. Email: bruno.cauli@upmc.fr

Edith Hamel:

Laboratory of Cerebrovascular Research, Montreal Neurological Institute, McGill University, Montréal, Québec H3A 2B4, Canada.

Email: edith.hamel@mcgill.ca

Key words: Astrocyte, cerebral blood flow, neurovascular coupling, functional hyperemia, neuronal activity, hemodynamic signals.

\section{Abstract (77 words):}

How can blood rapidly and precisely reach active neurons at a given time and location has remained enigmatic for a long time. A 2003 paper by Zonta et al. suggested key roles for astrocytes in the signaling between neurons and blood vessels. While a consensus on the specific intermediary roles of astrocytes in this process is still evolving, research in the past 15 years has led to a deeper and more refined understanding of the "neuro-glio-vascular" unit.

\section{Main text:}

The brain is an extremely energy consuming organ that depends on constant glucose and oxygen supply from the blood. The relationship between neuronal activity and local increases in cerebral blood flow, referred to as neurovascular coupling (NVC), was recognized more than a century ago. It is generally considered so precise that modern brain imaging techniques have used changes in hemodynamic signals to map neuronal activity for decades despite limited knowledge of the underlying mechanisms. But how do neurons signal to blood vessels about changes in local energy demands?

The era before Zonta et al.'s paper: Anatomical investigations on the relationship between neurons and local brain microvessels led to the concept of the neuronal-astrocytic-vascular functional unit, currently known as the "neurovascular unit". It rapidly became clear that astrocytes are not a mere decoration of this unit but exert key roles as intermediaries in the dialogue between neurons and blood vessels. However, it was not until 1997 that Alkayed and colleagues [1] convincingly showed that inhibiting the formation of astroglial P-450 epoxygenase-derived arachidonic acid (AA) products, likely epoxyeicosatrienoic acids (EETs), decreased the glutamate-induced blood flow response in the rat cerebral cortex. At that time, 
studies relied mainly on pharmacological approaches at a mesoscopic level, and NVC had been linked to both neuronal and astroglial messengers. This period also corresponded to the emergence of novel imaging techniques and tools, opening a new era for NVC investigations both in vitro and in vivo.

The advent of Zonta et al.'s paper: In 2003, a study by Zonta, Angulo and colleagues [2] in the laboratory of Prof Carmignoto in Padova, Italy, transformed NVC research. Using an array of methodologies - acute rat brain slices, confocal imaging of calcium $\left(\mathrm{Ca}^{2+}\right)$ dynamics, blood vessel imaging, neuronal or astroglial stimulation and pharmacological manipulations, together with in vivo cerebral blood flow measurements following forepaw stimulation - the authors provided the first demonstration that neuron to astrocyte signaling is central to NVC and revealed some of the key steps in this process. They found that glutamate, via mGluR group I, triggered $\mathrm{Ca}^{2+}$ increases in astrocyte somata and perivascular endfeet. Astrocytic $\mathrm{Ca}^{2+}$ signals temporally correlated with arteriolar dilation, and a cyclooxygenase (COX)-derivative of AA, possibly prostaglandin E2 ( $\mathrm{PGE}_{2}$ ), was involved in the response. In addition, the authors showed that stimulating an individual astrocyte, by touching or by applying intracellular depolarizing currents via a patch pipette, suffices to cause nearby arteriole dilation. The paper led to new research endeavors to untangle the role of astrocytes in controlling microvascular tone, and influenced the field of NVC research in the years to come.

\section{The era following Zonta et al.'s paper:}

The early consensus: Concurrent and follow-up studies in rodent acute cortical slices in young animals used electrical stimulation and/or 2-photon $\mathrm{Ca}^{2+}$ uncaging to increase intracellular $\mathrm{Ca}^{2+}$ signaling in astrocyte somata, processes, or endfeet around brain arterioles. Their results confirmed Zonta et al.'s finding that $\mathrm{Ca}^{2+}$ increases in astrocytic endfeet transduce neuronal activity into arteriolar dilation. These studies further refined the picture on factors involved in this process. Specifically, they pointed towards involvement of inositol trisphosphate receptor (IP3R)-mediated internal $\mathrm{Ca}^{2+}$ release, and the production of AA derivatives via activation of phospholipase A2 (PLA2). Additionally, it was discovered that $\mathrm{Ca}^{2+}$ increases in astroglial endfeet could also induce arteriolar constriction. The response polarity (i.e., dilation vs. constriction) depended on various determinants including metabolic factors (pO2, lactate, adenosine, glucose), arteriolar resting tone and, particularly, nitric oxide (NO) levels that affect the production of EETs and of the contractile AA derivative 20-hydroxyeicosatetraenoic acid (20-HETE)[3, 4]. Further, activation of large conductance $\mathrm{Ca}^{2+}$-activated $\mathrm{K}^{+}$channels (BK channels) in perivascular astroglial endfeet was shown to increase external $\mathrm{K}^{+}$levels, with $\mathrm{K}^{+}$ then acting on inward-rectifier $\mathrm{K}^{+}(\mathrm{Kir})$ channels on vascular smooth muscle cells to dilate arterioles. Yet, strong astroglial $\mathrm{Ca}^{2+}$ increases resulted in massive $\mathrm{K}^{+}$release and large depolarization, which were found to induce vasoconstriction [5].

Parallel in vivo investigations employing photolysis of caged $\mathrm{Ca}^{2+}$ in astrocytic endfeet or $\mathrm{Ca}^{2+}$ imaging during sensory stimulations, confirmed the ability of astrocytes to respond to changes in neuronal activity to promptly $(0.5$ to $\sim 5 \mathrm{sec})$ dilate microarterioles, via PLA2 and COX-1 sensitive mechanisms [6].

The ensuing dilemma and controversies: Roughly a decade after the publication of Zonta et al.'s paper, a series of studies questioned some of the key assumptions pertaining to astrocytic involvement in NVC. One of the reservations related to the timing or extent of the astroglial $\mathrm{Ca}^{2+}$ responses - arguing that these responses are either too slow or too small to initiate the NVC 
response. This argument was partly counteracted through technical advances that allowed to confirm the existence of fast stimulus-evoked $\mathrm{Ca}^{2+}$ increases in astroglial endfeet that precede the hemodynamic responses [7]. Another reservation was on the involvement of the mGluRphospholipase C (PLC)-IP3R signaling pathway in the NVC response. It was suggested that mGluR5-induced $\mathrm{Ca}^{2+}$ increases in astrocytes are unlikely to regulate blood flow in the adult brain, as mGluR5 mRNA downregulates to barely detectable levels by the third postnatal week (in rodents), when most studies in slices were performed [8]. Accordingly, in adult IP3R(2) KO mice, physiological stimulation failed to induce $\mathrm{Ca}^{2+}$ rises in astroglial endfeet, while NVC responses remained intact. Further, selective DREADD (designer receptors exclusively activated by designer drugs) activation of astrocytes, mimicking Gq-GPCR-linked IP3R-dependent $\mathrm{Ca}^{2+}$ signaling, had no effect on basal cortical blood flow [9, 10]. Collectively, these findings questioned the degree of astrocytic involvement in NVC, at least in some contexts, and fuelled debates that inspired a new wave of investigations to resolve these discrepancies.

Towards a unifying mechanism: An important aspect to consider in relation to NVC is the branching level of blood vessels that any specific proposed mechanism acts on. As illustrated in Figure 1, pial arteries (top) branch into arterioles that penetrate the brain tissue, and which in turn branch into smaller capillaries. Could the controversies outlined above relate, at least in part, to divergent mechanisms acting at the arteriole vs. capillary level? At about the same time as the studies mentioned in the previous section, brain capillaries were found to often dilate before arterioles, and capillary dilation was suggested to account for the bulk of the blood flow increase following sensory stimulation [11]. The capillary response was argued to be mediated, in part, via activity-dependent $\mathrm{PGE}_{2}$ release and pericyte relaxation, although later studies questioned pericytes' involvement [12]. While components of the picture continue to be contested, a possible approach towards as unifying model is the appreciation that in the broader picture, NVC likely encompasses a variety of mechanisms, acting via different mediators and possibly at different levels of the vascular tree (Figure 1). Along this line, a rise in astrocyte $\left[\mathrm{Ca}^{2+}\right]_{\mathrm{i}}$ was found necessary for neurally-evoked capillary, but not arteriole, dilation to occur [13]. This process was associated with increased glutamatergic synaptic activity and ATP released from post-synaptic neurons acting on the P2X1 subunit of astroglial ATP-gated ion channels to increase $\mathrm{Ca}^{2+}$ entry from the extracellular space. This resulted in AA formation through phospholipase D2 (PLD2), COX-1 dependent synthesis and release of PGE 2 , presumably acting on EP4 receptors on pericytes, to dilate capillaries. A recent study [14] also showed that $\mathrm{K}^{+}$ generated by neuronal activity activates Kir channels (Kir2.1) in endothelial cells of brain capillaries that electrically propagate a retrograde hyperpolarization signal, thereby leading to dilation of upstream arterioles and increased perfusion in the capillary bed. This reveals that functional hyperemia can be initiated at the capillary level by multiple messengers, produced by different cell types, and that endothelial cells are key players in signaling to upstream arterioles. In addition, intraparenchymal arterioles have their own mechanisms of vasodilation which include, but are not limited to, NMDA receptor-mediated production and release of NO [13], likely from interneurons, and COX-2 derived $\mathrm{PGE}_{2}$ from pyramidal cells [15] (Figure 1). Such a multiplicity of NVC mechanisms acting at different levels of the vascular tree implies a fine and coordinated interplay between the different cell types of the "neuro-glio-vascular" unit, in which astrocytes do not necessarily initiate the NVC response. Further, such organization confers robustness to the neurovascular response as it provides multiple safety mechanisms. 
Overall influence on the field and remaining questions: Due to space constraints, we limited our comments to the cerebral cortex and excluded relevant studies on astroglial signaling in NVC in other brain regions. While Zonta et al.'s paper unraveled a role for neuron-to-astrocyte signaling in NVC that was substantially refined over the past 15 years, several open questions remain. Particularly, there is a need for a better understanding of $\boldsymbol{i}$ ) the age-dependent switch in astroglial receptors and the impact on NVC responses in development, ii) the respective contribution of arteriole and capillary responses that use astrocytes as intermediaries as opposed to direct vascular interactions, taking into account retrograde dilation, iii) the role of neurons and their vasoactive messengers in astroglial and vascular responses, iv) the involvement of other ion channels, such as TRPV4 channels, v) the role of resting, intrinsic astrocytic $\mathrm{Ca}^{2+}$ in hemodynamic regulation, vi) the reverse dialog, namely how changes in microvascular tone affect neuronal activity and, finally vii) the reliability of hemodynamic signals to map changes in neuronal activity in diseases that affect multiple components of the "neuro-glio-vascular" unit. These will lead, no doubt, to many more years of exciting new research on the cellular mechanisms underlying NVC in health and disease, and on how astrocytic signaling intervenes at rest and during activation states.

Acknowledgments: We thank Drs. Hélène Girouard (Dept de Pharmacologie, Université de Montréal, QC, Canada), Dongong Li (NPS-IBPS, Paris, France), Maria LaCalle-Aurioles, Miled Bourourou and Alexis Machado (Montreal Neurological Institute, McGill University, Montréal, QC, Canada), as well as Lianne Trigiani and Jessika Royea (Montreal Neurological Institute, McGill University) for their helpful comments on the manuscript. BC is supported by a grant from the Agence Nationale de la Recherche (ANR-17-CE37-0010-03) and EH by the Canadian Institute of Health Research (CIHR, MOP-142417). 


\section{References:}

1. Alkayed, N.J. et al. (1997) Role of P-450 arachiconic acid epoxygenase in the response of cerebral blood flow to glutamate in rats. Stroke 28, 1066-1072.

2. Zonta, M. et al. (2003) Neuron-to-astrocyte signaling is central to the dynamic control of brain microcirculation. Nature Neurosci. 6 (1), 43-50.

3. Gordon, G.R. et al. (2008) Brain metabolism dictates the polarity of astrocyte control over arterioles. Nature 456 (7223), 745-9.

4. Blanco, V.M. et al. (2008) Tone-dependent vascular responses to astrocyte-derived signals. Am J Physiol Heart Circ Physiol 294 (6), H2855-63.

5. Girouard, H. et al. (2010) Astrocytic endfoot Ca2+ and BK channels determine both arteriolar dilation and constriction. Proc Natl Acad Sci U S A 107 (8), 3811-6.

6. Takano, T. et al. (2006) Astrocyte-mediated control of cerebral blood flow. Nat Neurosci 9 (2), 260-7.

7. Lind, B.L. et al. (2013) Rapid stimulus-evoked astrocyte Ca2+ elevations and hemodynamic responses in mouse somatosensory cortex in vivo. Proc Natl Acad Sci U S A 110 (48), E4678-87.

8. Sun, W. et al. (2013) Glutamate-dependent neuroglial calcium signaling differs between young and adult brain. Science 339 (6116), 197-200.

9. Nizar, K. et al. (2013) In vivo stimulus-induced vasodilation occurs without IP3 receptor activation and may precede astrocytic calcium increase. J Neurosci 33 (19), 8411-22.

10. Bonder, D.E. and McCarthy, K.D. (2014) Astrocytic Gq-GPCR-linked IP3R-dependent Ca2+ signaling does not mediate neurovascular coupling in mouse visual cortex in vivo. J Neurosci 34 (39), 13139-50.

11. Hall, C.N. et al. (2014) Capillary pericytes regulate cerebral blood flow in health and disease. Nature 508 (7494), 55-60.

12. Hill, R.A. et al. (2015) Regional Blood Flow in the Normal and Ischemic Brain Is Controlled by Arteriolar Smooth Muscle Cell Contractility and Not by Capillary Pericytes. Neuron 87 (1), 95110.

13. Mishra, A. et al. (2016) Astrocytes mediate neurovascular signaling to capillary pericytes but not to arterioles. Nat Neurosci 19 (12), 1619-1627.

14. Longden, T.A. et al. (2017) Capillary $K(+)$-sensing initiates retrograde hyperpolarization to increase local cerebral blood flow. Nat Neurosci 20 (5), 717-726.

15. Lacroix, A. et al. (2015) COX-2-Derived Prostaglandin E2 Produced by Pyramidal Neurons Contributes to Neurovascular Coupling in the Rodent Cerebral Cortex. J Neurosci 35 (34), 11791-810. 
Figure legend:

Proposed unifying model of neurovascular coupling. Top left: Glutamate released by active neurons activates astrocytic metabotropic glutamate receptor (mGluR) leading to an intracellular $\left[\mathrm{Ca}^{2+}\right]$ surge from internal stores in astrocytic endfeet, and subsequent activation of phospholipase A2 (PLA2) and large conductance $\mathrm{Ca}^{2+}$-activated $\mathrm{K}^{+}$(BK) channels. PLA2 produces arachidonic acid (AA) from membrane phospholipids (MPL) which can be converted into the vasodilatory prostaglandin $\mathrm{E}_{2}\left(\mathrm{PGE}_{2}\right)$ and epoxyeicosatrienoic acids (EETs), via cyclooxygenase (COX)-1 and cytochrome P450 epoxyganase (CYP) pathways, respectively. $\mathrm{PGE}_{2}$ and EETs diffuse into the extracellular space and activate smooth muscle cell type $4 \mathrm{PGE}_{2}$ (EP4) and undetermined EETs receptors to elicit arteriolar dilation. BK channels activation induces $\mathrm{K}^{+}$release into the extracellular space leading to a hyperpolarization of smooth muscle cells via inwardly rectifying $\mathrm{K}^{+}$(Kir) channels, resulting in dilation of arterioles. Additionally, ATP produced by neuronal activity activates astrocytic ionotropic P2X1-containing ATP receptors leading to an intracellular $\left[\mathrm{Ca}^{2+}\right]$ influx in astrocytic endfeet from the extracellular space. This leads to the activation of phospholipase D2 (PLD2), and production of AA and PGE 2 via the COX-1 pathway in the astrocyte. Astroglial release of $\mathrm{PGE}_{2}$ would then dilate capillaries, possibly by acting on EP4 receptors on pericytes and/or via other mechanisms. Bottom left: Neuronal activity causes an increase in extracellular $\mathrm{K}^{+}$- either directly, or indirectly via astrocytes - which in turn hyperpolarizes capillary endothelial cells via Kir channels. This hyperpolarization travels upstream along the vasculature through gap junctions (Cnx), leading to the relaxation of vascular smooth muscle cells and arteriole dilation. Right: Glutamate activates NMDA receptors (NDMA-R) on pyramidal cells or on nitric oxide synthase (NOS) interneurons, leading to an increase of intracellular $\left[\mathrm{Ca}^{2+}\right]$. In pyramidal cells, this leads to the production and release of $\mathrm{PGE}_{2}$ via the COX-2 pathway. Activation of type $2 \mathrm{PGE}_{2}$ (EP2) and EP4 receptors relaxes smooth muscle cells and dilates arterioles. In NOS interneurons, the increase of intracellular $\left[\mathrm{Ca}^{2+}\right]$ activates NOS, producing NO from arginine (ARG) which dilates arterioles. Plain and dashed arrows denote single and multiple steps biosynthetic pathways, respectively. Within the vessel lumen, open arrows indicate the direction of blood flow and twisted arrows the retrograde hyperpolarization signal along the endothelial cells. Inspired from Figure S1[13]. 


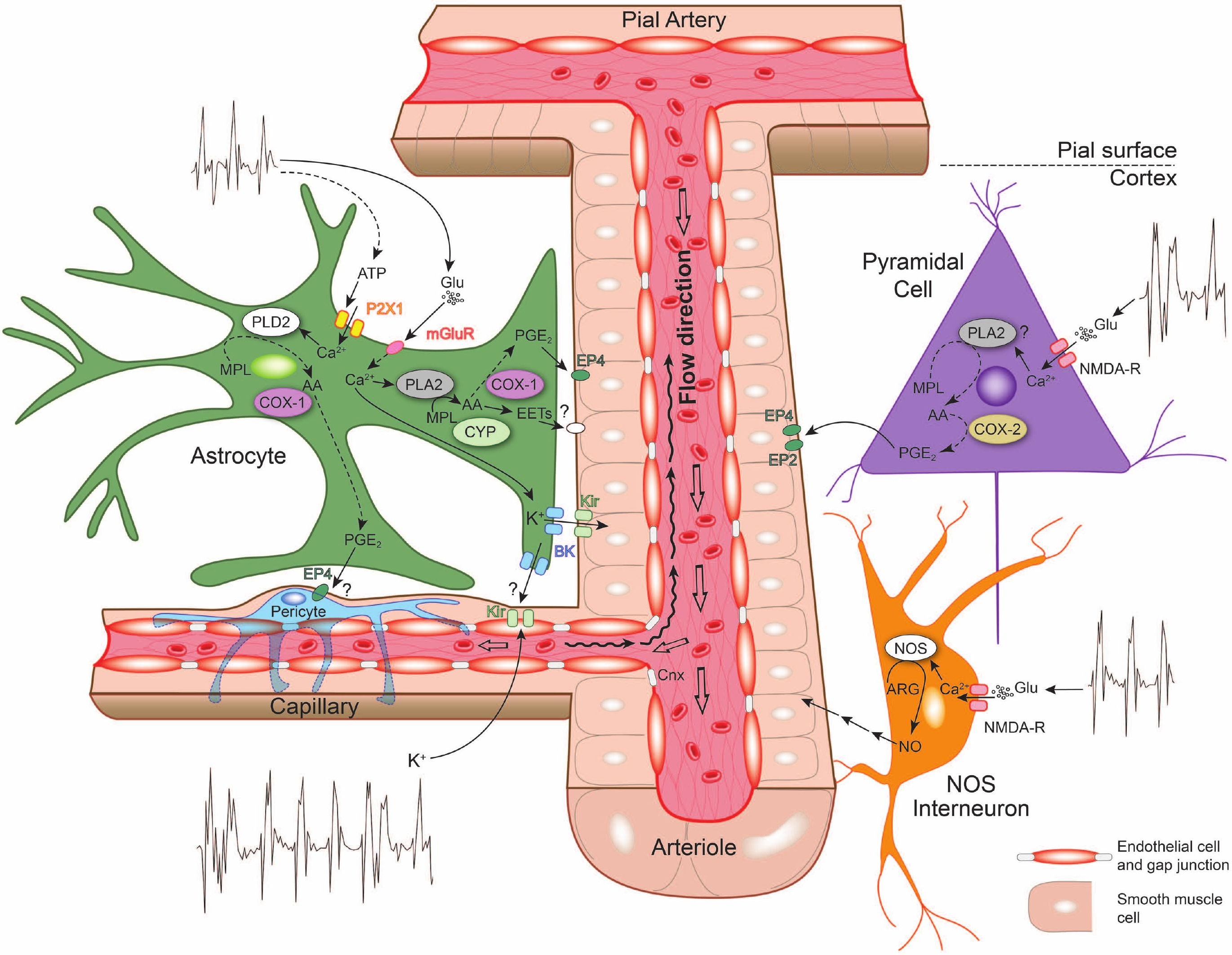

